\title{
Integral Representation of One Class of Entire Functions
}

\author{
R. V. Khats' \\ Abstract. In this paper, we study an integral representation of \\ one class of entire functions. Conditions for the existence of this \\ representation in terms of certain solutions of some differential \\ equations are found. We obtain asymptotic estimates of entire \\ functions from the considered class of functions. We also give \\ examples of entire functions from this class.
}

Key Words: Paley-Wiener theorem, entire function of exponential type, differential equation, Schwarz inequality, asymptotic estimate

Mathematics Subject Classification 2010: 30D10, 30D20, 30E10, 30E15, 42A10, 42B10, 44A05, 44A15

\section{Introduction}

Let $L^{p}(X)$ be the space of all measurable functions $f: X \rightarrow \mathbb{C}$ on a measurable set $X \subseteq \mathbb{R}$ with the norm

$$
\|f\|_{L^{p}(X)}^{p}:=\int_{X}|f(x)|^{p} d x, \quad 1 \leq p<+\infty .
$$

An entire function $G$ is said to be of exponential type $\sigma \in[0 ;+\infty)$ ( [3, p. 4], [14, p. 12]) if for any $\varepsilon>0$, there exists a constant $c(\varepsilon)$ such that

$$
|G(z)| \leq c(\varepsilon) \exp ((\sigma+\varepsilon)|z|)
$$

for all $z \in \mathbb{C}$.

Denote by $P W_{\sigma}^{2}$ the set of all entire functions of exponential type $\sigma$ whose narrowing on $\mathbb{R}$ belongs to the space $L^{2}(\mathbb{R})$, and by $P W_{\sigma,+}^{2}$ denote the class of even entire functions from $P W_{\sigma}^{2}$. According to the Paley-Wiener theorem (see, for example, [3, p. 69], [5, p. 4263], [14, p. 13]), the class $P W_{\sigma}^{2}$ coincides with the class of functions $G$ admitting the representation

$$
G(z)=\int_{-\sigma}^{\sigma} e^{i t z} g(t) d t, \quad g \in L^{2}(-\sigma ; \sigma)
$$


and the class $P W_{\sigma,+}^{2}$ consists of the functions $G$ representable in the form

$$
G(z)=\int_{0}^{\sigma} \cos (t z) g(t) d t, \quad g \in L^{2}(0 ; \sigma) .
$$

Moreover, $\|g\|_{L^{2}(0 ; \sigma)}=\sqrt{2 / \pi}\|G\|_{L^{2}(0 ;+\infty)}$ and

$$
g(t)=\frac{2}{\pi} \int_{0}^{+\infty} G(z) \cos (t z) d z
$$

The problem of integral representations of various classes of entire functions has been studied in many papers (see, for instance, $[1,3-12,14]$ ). In particular, in [7] (see also 812$]$ ), it was described the class $\widehat{\mathcal{E}}$ of entire functions $G$ which can be presented in the form

$$
G(z)=\int_{0}^{1}(\cos (t z)+t z \sin (t z)) g(t) d t, \quad g \in L^{2}(0 ; 1) .
$$

Denote by $\mathcal{E}$ the class of even entire functions of exponential type $\sigma \leq 1$ for which $w^{-2}(G(w)-G(0))$ belongs to $L^{1}(\mathbb{R}), z \int_{z}^{+\infty} w^{-2} G(w) d w$ belongs to $L^{2}(0 ;+\infty)$, and $\int_{-\infty}^{+\infty} w^{-2}(G(w)-G(0)) d w=0$.

Theorem A. ([7, p. 11]) The following conditions are equivalent:

1) $G \in \widehat{\mathcal{E}}$

2) $G \in \mathcal{E}$

3) on $(0 ;+\infty)$, the equation $f(z)-z f^{\prime}(z)=G(z)$ has a solution $f=F$ belonging to $P W_{1,+}^{2}$;

4) $G$ is an even entire function, and $\widetilde{G}(z):=G(z)-z \int_{0}^{z} w^{-1} G^{\prime}(w) d w$ belongs to the space $P W_{1,+}^{2}$;

5) $G$ is an even entire function of exponential type $\sigma \leq 1, w^{-2}(G(w)-$ $G(0))$ belongs to $L^{1}(\mathbb{R}), z \int_{z}^{+\infty} w^{-2} G(w) d w$ belongs to $L^{2}(0 ;+\infty)$, and $G(z)=$ $G_{1}(z)+G_{1}(-z)$, where $G_{1}$ is an entire function satisfying $\left|G_{1}(z)\right| \leq c_{1}(1+$ $|z|) / \sqrt{1+\operatorname{Im} z}, c_{1}>0, \operatorname{Im} z \geq 0$.

The aim of the present paper is to give a description of the class $\widetilde{\mathcal{E}}$ of entire functions $Q$ which can be presented in the form

$$
Q(z)=\int_{0}^{1}\left(-z^{2} t^{2} \cos (t z)+3 t z \sin (t z)+3 \cos (t z)\right) g(t) d t, \quad g \in L^{2}(0 ; 1) .
$$

Such a class $\widetilde{\mathcal{E}}$ arises in the investigation of some boundary-value problems ( [11], [12]), whose singularity lies in the fact that the set of their canonical eigenfunctions can be overflowed. We obtain an analog of Theorem A for this class of functions. This result is contained in Theorems 1 3. Close assertions can be found in [4], [6]. 


\section{Main results}

Our principal results are the following statements.

Theorem 1 An entire function $Q$ has the representation (1) if and only if the differential equation

$$
-z f^{\prime}(z)+3 f(z)=Q(z)
$$

has a solution $f=G$ belonging to $\mathcal{E}$.

Proof. Necessity. Let $Q$ have the representation (1) and

$$
G(z)=\int_{0}^{1}(\cos (t z)+t z \sin (t z)) g(t) d t, \quad g \in L^{2}(0 ; 1) .
$$

By Theorem A, we have $G \in \mathcal{E}$ and

$$
-z G^{\prime}(z)+3 G(z)=\int_{0}^{1}\left(-z^{2} t^{2} \cos (t z)+3 t z \sin (t z)+3 \cos (t z)\right) g(t) d t=Q(z) .
$$

Therefore, the necessity has been proved.

Sufficiency. If $f=G$ is the indicated solution to the differential equation (2), then according to Theorem A, we have

$$
f(z)=\int_{0}^{1}(\cos (t z)+t z \sin (t z)) g(t) d t, \quad g \in L^{2}(0 ; 1) .
$$

Hence,

$Q(z)=-z f^{\prime}(z)+3 f(z)=\int_{0}^{1}\left(-z^{2} t^{2} \cos (t z)+3 t z \sin (t z)+3 \cos (t z)\right) g(t) d t$.

Theorem 1 is proved.

Remark 1 Let

$$
J_{\nu}(z)=\sum_{k=0}^{\infty} \frac{(-1)^{k}(z / 2)^{\nu+2 k}}{k ! \Gamma(\nu+k+1)}
$$

be a Bessel function of the first kind of index $\nu \in \mathbb{R}$, where $\Gamma$ is the gamma function. Since (see [13, p. 55])

$$
z^{2} \sqrt{t z} J_{-5 / 2}(t z)=\sqrt{\frac{2}{\pi}} \frac{-z^{2} t^{2} \cos (t z)+3 t z \sin (t z)+3 \cos (t z)}{t^{2}},
$$

we obtain that the function $Q \in \widetilde{\mathcal{E}}$ can be represented in the form

$$
Q(z)=\sqrt{\frac{\pi}{2}} \int_{0}^{1} z^{2} t^{2} \sqrt{t z} J_{-5 / 2}(t z) g(t) d t, \quad g \in L^{2}(0 ; 1) .
$$


Example 1 Let $\alpha:=4(-\pi+2) / \pi^{3}$. The function

$$
\begin{aligned}
Q(z) & =z \frac{\left(1-\alpha\left(z^{2}-\pi^{2} / 4\right)\right)\left(z^{2}-\pi^{2} / 4\right) \sin z+2 z \cos z}{\left(z^{2}-\pi^{2} / 4\right)^{2}} \\
& +3 \frac{\cos z}{z^{2}-\pi^{2} / 4}\left(1-\alpha\left(z^{2}-\pi^{2} / 4\right)\right)
\end{aligned}
$$

belongs to $\widetilde{\mathcal{E}}$ with

$$
g(t)=\frac{1}{t^{2} \pi^{3}}\left(8-8 \cos \left(\frac{\pi}{2} t\right)-4 \pi t \sin \left(\frac{\pi}{2} t\right)\right) .
$$

Indeed, the function

$$
f(z)=\frac{\cos z}{z^{2}-\pi^{2} / 4}\left(1-\alpha\left(z^{2}-\pi^{2} / 4\right)\right)
$$

belongs to $\widehat{\mathcal{E}}$ (see [7, Example 2, p. 13]) and is a solution to the equation (2), because

$$
f^{\prime}(z)=\frac{-\left(z^{2}-\pi^{2} / 4\right)\left(1-\alpha\left(z^{2}-\pi^{2} / 4\right)\right) \sin z-2 z \cos z}{\left(z^{2}-\pi^{2} / 4\right)^{2}} .
$$

Therefore, according to Theorems $A$ and 1 , the function $Q$ admits representation (1).

Theorem 2 An entire function $Q$ has the representation (1) if and only if the differential equation

$$
z^{2} f^{\prime \prime}(z)-3 z f^{\prime}(z)+3 f(z)=Q(z)
$$

has a solution $f=F$ belonging to $P W_{1,+}^{2}$. In this case, the function $z^{-1}\left(z^{-1} Q^{\prime}(z)\right)^{\prime}$ also belongs to the space $P W_{1,+}^{2}$ and $g$ can be found by one of the following formulas

$$
\begin{gathered}
g(t)=\frac{2}{\pi} \int_{0}^{+\infty} F(z) \cos (t z) d z, \\
g(t)=\frac{2}{\pi t^{4}} \int_{0}^{+\infty} \frac{1}{z}\left(\frac{Q^{\prime}(z)}{z}\right)^{\prime} \cos (t z) d z .
\end{gathered}
$$

Proof. Necessity. Let the function $Q$ can be presented in the form (1) and

$$
F(z)=\int_{0}^{1} \cos (t z) g(t) d t, \quad g \in L^{2}(0 ; 1) .
$$

Then

$$
Q^{\prime}(z)=\int_{0}^{1}\left(z^{2} t^{3} \sin (t z)+t^{2} z \cos (t z)\right) g(t) d t, \quad g \in L^{2}(0 ; 1), \quad Q^{\prime}(0)=0,
$$




$$
\begin{gathered}
\frac{Q^{\prime}(z)}{z}=\int_{0}^{1}\left(z t^{3} \sin (t z)+t^{2} \cos (t z)\right) g(t) d t \\
\frac{1}{z}\left(\frac{Q^{\prime}(z)}{z}\right)^{\prime}=\int_{0}^{1} t^{4} \cos (t z) g(t) d t .
\end{gathered}
$$

Due to the Paley-Wiener theorem, the functions $F(z)$ and $z^{-1}\left(z^{-1} Q^{\prime}(z)\right)^{\prime}$ belong to $P W_{1,+}^{2}$. Moreover,

$$
F^{\prime}(z)=-\int_{0}^{1} t \sin (t z) g(t) d t, \quad F^{\prime \prime}(z)=-\int_{0}^{1} t^{2} \cos (t z) g(t) d t
$$

and

$$
\begin{aligned}
z^{2} F^{\prime \prime}(z) & -3 z F^{\prime}(z)+3 F(z) \\
& =\int_{0}^{1}\left(-z^{2} t^{2} \cos (t z)+3 t z \sin (t z)+3 \cos (t z)\right) g(t) d t=Q(z) .
\end{aligned}
$$

Therefore, the necessity has been proved.

Sufficiency. Let the equation (3) has a solution $f=F$ belonging to $P W_{1,+}^{2}$. Then in accordance with the Paley-Wiener theorem,

$$
f(z)=\int_{0}^{1} \cos (t z) g(t) d t, \quad g \in L^{2}(0 ; 1) .
$$

Hence,

$$
\begin{aligned}
Q(z) & =z^{2} f^{\prime \prime}(z)-3 z f^{\prime}(z)+3 f(z) \\
& =\int_{0}^{1}\left(-z^{2} t^{2} \cos (t z)+3 t z \sin (t z)+3 \cos (t z)\right) g(t) d t,
\end{aligned}
$$

and we obtain representation (1). Formulas (4) and (5) follow from the equalities (6) and (7) and the formula for the inverse Fourier cosine-transformation. Theorem 2 is proved.

Example 2 The function $Q(z)=2 z^{4} \cos z$ does not belong to $\widetilde{\mathcal{E}}$. In fact, for this function $Q$ the differential equation (3) has a solution $F(z)=C_{1} z+$ $C_{2} z^{3}-2 z^{2} \cos z+2 z \sin z$. But there are no constants $C_{1}$ and $C_{2}$ for which the function $F$ belongs to $P W_{1,+}^{2}$. Indeed, $F$ is an even entire function only if $C_{1}=C_{2}=0$, and in this case, the function $G(z)=-2 z^{2} \cos z+2 z \sin z$ does not belong to $W_{1,+}^{2}$ since $G \notin L^{2}(\mathbb{R})$. Hence, the equation (3) with $Q(z)=2 z^{4} \cos z$ has no solution belonging to $P W_{1,+}^{2}$. Thus, according to Theorem 2, the function $Q$ cannot be represented in the form (1).

Remark 2 Theorems 1 and 2 give a description of functions $Q$, for which differential equations (2) and (3) have solutions in the corresponding spaces. Similar problems are considered in many investigations (see, for example, [2]). 
Theorem 3 Let an entire function $Q \in \widetilde{\mathcal{E}}$ be defined by the formula (1). Then

$$
|Q(z)| \leq c_{3} \frac{e^{|\operatorname{Im} z|}}{\sqrt{1+|\operatorname{Im} z|}}(1+|z|)^{2}, \quad z \in \mathbb{C}, \quad c_{3}>0
$$

and $Q$ is an even entire function of exponential type $\sigma \leq 1$. Moreover, $w^{-4} Q(w)$ belongs to $L^{1}(1 ;+\infty)$, and $Q(z)=Q_{1}(z)+Q_{1}(-z)$ where $Q_{1}$ is an entire function satisfying

$$
\left|Q_{1}(z)\right| \leq c_{4} \frac{(1+|z|)^{2}}{\sqrt{1+\operatorname{Im} z}}, \quad \operatorname{Im} z \geq 0, \quad c_{4}>0 .
$$

Proof. Indeed, let $Q \in \widetilde{\mathcal{E}}$ and $Q(z):=F_{1}(z)+F_{2}(z)+F_{3}(z)$ where

$$
\begin{gathered}
F_{1}(z)=-z^{2} \int_{0}^{1} t^{2} \cos (t z) g(t) d t, \quad F_{2}(z)=3 z \int_{0}^{1} t \sin (t z) g(t) d t, \\
F_{3}(z)=3 \int_{0}^{1} \cos (t z) g(t) d t, \quad g \in L^{2}(0 ; 1) .
\end{gathered}
$$

Due to the Paley-Wiener theorem, the functions $F_{1}(z) / z^{2}, F_{2}(z) / z$ and $F_{3}(z)$ belong to the space $P W_{1}^{2}$, and

$$
F_{3}(z)=3 \int_{0}^{1} \frac{e^{i t z}}{2} g(t) d t+3 \int_{0}^{1} \frac{e^{-i t z}}{2} g(t) d t, \quad g \in L^{2}(0 ; 1) .
$$

A simple estimate with the use of the Schwarz inequality shows that

$$
|Q(z)| \leq c_{3} \frac{e^{|\operatorname{Im} z|}}{\sqrt{1+|\operatorname{Im} z|}}(1+|z|)^{2}, \quad z \in \mathbb{C}, \quad c_{3}>0 .
$$

Hence, $Q$ is an even entire function of exponential type $\sigma \leq 1$. Further,

$$
\begin{aligned}
\int_{x}^{+\infty} & \left|\frac{Q(w)}{w^{2}}\right|^{2} d w \\
& \leq 3 \int_{x}^{+\infty}\left(\left|\frac{F_{1}(w)}{w^{2}}\right|^{2}+\left|\frac{F_{2}(w)}{w^{2}}\right|^{2}+\left|\frac{F_{3}(w)}{w^{2}}\right|^{2}\right) d w<+\infty
\end{aligned}
$$

and from the Schwarz inequality we obtain

$$
\left|\int_{x}^{+\infty} \frac{Q(w)}{w^{4}} d w\right| \leq \frac{1}{x \sqrt{3 x}}\left(\int_{x}^{+\infty}\left|\frac{Q(w)}{w^{2}}\right|^{2} d w\right)^{1 / 2}<+\infty, \quad x \in(0 ;+\infty) .
$$

Therefore, $w^{-4} Q(w)$ belongs to $L^{1}(1 ;+\infty)$. Furthermore, $Q(z)=Q_{1}(z)+$ $Q_{2}(z)$, where

$$
Q_{1}(z):=\int_{0}^{1}\left(-z^{2} t^{2} \frac{e^{i t z}}{2}+3 z t \frac{e^{i t z}}{2 i}+3 \frac{e^{i t z}}{2}\right) g(t) d t
$$




$$
Q_{2}(z):=\int_{0}^{1}\left(-z^{2} t^{2} \frac{e^{-i t z}}{2}-3 z t \frac{e^{-i t z}}{2 i}+3 \frac{e^{-i t z}}{2}\right) g(t) d t .
$$

Here $Q_{2}(z)=Q_{1}(-z)$, and using Schwarz inequality, we get

$$
\begin{aligned}
\left|Q_{1}(z)\right| & \leq \frac{3}{2}(1+|z|)^{2}\|g\|_{L^{2}(0 ; 1)}\left(\int_{0}^{1} e^{-2 t y} d t\right)^{1 / 2} \\
& =\frac{3}{2}(1+|z|)^{2}\|g\|_{L^{2}(0 ; 1)}\left(\frac{1-e^{-2 y}}{2 y}\right)^{1 / 2} \leq c_{4} \frac{(1+|z|)^{2}}{\sqrt{1+\operatorname{Im} z}}
\end{aligned}
$$

for $z=x+i y$ and $y \geq 0$, where $c_{4}>0$. Theorem 3 is proved.

Corollary 1 If a function $Q$ belongs to $\widetilde{\mathcal{E}}$, then

$$
\int_{x}^{+\infty}\left|\frac{Q(w)}{w^{2}}\right|^{2} d w<+\infty, \quad \int_{x}^{+\infty} \frac{|Q(w)|}{w^{4}} d w<+\infty
$$

for each $x \in(0 ;+\infty)$, and

$$
\left|\int_{x}^{+\infty} \frac{Q(w)}{w^{4}} d w\right|=o\left(\frac{1}{x \sqrt{x}}\right) \text { as } x \rightarrow+\infty .
$$

\section{Conclusions}

The paper is devoted to the study of an integral representation of the class $\widetilde{\mathcal{E}}$ of entire functions. The conditions for this representation in terms of the existence of certain solutions of some differential equations are found (see Theorems 1 and 2). Asymptotic estimates of entire functions $Q \in \widetilde{\mathcal{E}}$ are obtained (see Theorem 3). The corresponding examples are given. Those results can be used for the investigation of completeness and minimality of the trigonometric system $\left\{-t^{2} \rho_{k}^{2} \cos \left(t \rho_{k}\right)+3 t \rho_{k} \sin \left(t \rho_{k}\right)+3 \cos \left(t \rho_{k}\right): k \in \mathbb{N}\right\}$ in the space $L^{2}(0 ; 1)$, where $\left(\rho_{k}\right)_{k \in \mathbb{N}}$ is a sequence of distinct nonzero complex numbers.

\section{Acknowledgments}

The author is very grateful to the anonymous referees for their valuable remarks and suggestions toward the improvement of the paper.

\section{References}

[1] M.M. Dzhrbashyan, Integral transforms and representations of functions in the complex domain, Nauka, Moscow, 1966. (in Russian) 
[2] I. Laine, Nevanlinna theory and complex differential equations, Walter de Gruiter, Berlin, 2011. https://doi.org/10.1515/9783110863147

[3] B.Ya. Levin, Lectures on entire functions. Transl. Math. Monogr., Amer. Math. Soc., Providence, R.I., 150, 1996.

[4] V.I. Lutsenko and R.S. Yulmukhametov, Generalization of the PaleyWiener theorem in weighted spaces, Math. Notes, 48 (1990), no. 5, pp. 1131-1136. https://doi.org/10.1007/bf01236300

[5] A.M. Sedletskii, Analytic Fourier transforms and exponential approximations. I., J. Math. Sci., 129 (2005), no. 6, pp. 4251-4408. https://doi.org/10.1007/s10958-005-0349-y

[6] V.K. Tuan and A.I. Zayed, Paley-Wiener-type theorems for a class of integral transforms, J. Math. Anal. Appl., 266 (2002), no. 1, pp. 200226. https://doi.org/10.1006/jmaa.2001.7740

[7] B.V. Vynnyts'kyi and V.M. Dilnyi, On approximation properties of one trigonometric system, Russ. Math., 58 (2014), no. 11, pp. 10-21. https://doi.org/10.3103/s1066369x14110024

[8] B.V. Vynnyts'kyi and R.V. Khats', Some approximation properties of the systems of Bessel functions of index -3/2, Mat. Stud., 34 (2010), no. 2, pp. 152-159.

[9] B.V. Vynnyts'kyi and R.V. Khats', Completeness and minimality of systems of Bessel functions, Ufa Math. J., 5 (2013), no. 2, pp. 131-141. https://doi.org/10.13108/2013-5-2-131

[10] B.V. Vynnyts'kyi and R.V. Khats', On the completeness and minimality of sets of Bessel functions in weighted $L^{2}$-spaces, Eurasian Math. J., 6 (2015), no. 1, pp. 123-131.

[11] B.V. Vynnyts'kyi and O.V. Shavala, Boundedness of solutions of a second-order linear differential equation and a boundary value problem for Bessel's equation, Mat. Stud., 30 (2008), no. 1, pp. 31-41. (in Ukrainian)

[12] B.V. Vynnyts'kyi and O.V. Shavala, Some properties of boundary value problems for Bessel's equation, Math. Bull. Shevchenko Sci. Soc., 10 (2013), pp. 169-172.

[13] G.N. Watson, A treatise on the theory of Bessel functions, Cambridge University Press, Cambridge, 1944. 
[14] N. Wiener and R.C. Paley, Fourier transforms in the complex domain, USA: Amer. Math. Soc., Providence, R.I., 19, 1934.

Ruslan V. Khats'

Drohobych Ivan Franko State Pedagogical University,

Institute of Physics, Mathematics, Economics and Innovation Technologies, 3 Stryiska Str., 82100 Drohobych, Ukraine.

khats@ukr.net

Please, cite to this paper as published in

Armen. J. Math., V. 14, N. 1(2022), pp. 19

https://doi.org/10.52737/18291163-2022.14.1-1-9 